\title{
Specificity of insulin signalling in human skeletal muscle as revealed by small interfering RNA
}

\author{
A. Krook • J. R. Zierath
}

Received: 18 December 2008 / Accepted: 23 February 2009/Published online: 31 March 2009

(C) Springer-Verlag 2009

\begin{abstract}
Insulin action on metabolically active tissues is a complex process involving positive and negative feedback regulation to control whole body glucose homeostasis. At the cellular level, glucose and lipid metabolism, as well as protein synthesis, are controlled through canonical insulin signalling cascades. The discovery of small interfering RNA (siRNA) allows for the molecular dissection of critical components of the regulation of metabolic and gene regulatory events in insulin-sensitive tissues. The application of siRNA to tissues of human origin allows for the molecular dissection of the mechanism(s) regulating glucose and lipid metabolism. Penetration of the pathways controlling insulin action in human tissue may aid in discovery efforts to develop diabetes prevention and treatment strategies. This review will focus on the use of siRNA to validate critical regulators controlling insulin action in human skeletal muscle, a key organ important for the control of whole body insulin-mediated glucose uptake and metabolism.
\end{abstract}

Keywords Gene expression · Glucose metabolism · Insulin signalling $\cdot$ Lipid metabolism .

Protein phosphorylation · Lipid metabolism · siRNA . Skeletal muscle $\cdot$ Therapy $\cdot$ Target validation

\footnotetext{
A. Krook $\cdot$ J. R. Zierath $(\bowtie)$

Department of Molecular Medicine and Surgery,

Section of Integrative Physiology, Karolinska Institutet,

von Eulers väg 4, 4th Floor,

17177 Stockholm, Sweden

e-mail: Juleen.Zierath@ki.se
}

\footnotetext{
A. Krook $\cdot$ J. R. Zierath

Department of Physiology and Pharmacology,

Karolinska Institutet,

Stockholm, Sweden
}

\author{
Abbreviations \\ AS160 AKT substrate of $160 \mathrm{kDa}$ \\ JNK c-Jun $\mathrm{NH}_{2}$-terminal kinase \\ ERK1/2 Extracellular signal-regulated kinase 1/2 \\ IKK Inhibitor of nuclear factor- $\mathrm{KB}$ kinase \\ KRLB Kinase regulatory loop binding \\ MAPK Mitogen-activated protein kinase \\ MAP4K4 Mitogen-activated protein kinase kinase kinase \\ kinase 4 \\ NF- $k B \quad$ Nuclear factor $k B$ \\ p70S6K p70 Ribosomal S6 kinase, \\ PI3K Phosphatidylinositol 3-kinase \\ PTEN Phosphatase and tensin homologue \\ siRNA Small interfering RNA \\ mTOR Mammalian target of rapamycin \\ TNFR1 TNF- $\alpha$ receptor 1
}

\section{Insulin signalling and type 2 diabetes}

The complexity of the insulin signalling cascades has become clearer following the identification of critical components, or nodes, within the network that are essential mediators of insulin action [1] (Fig. 1). Insulin signalling via the cell-surface receptor is linked to the phosphorylation of IRSs [2]. At this critical juncture, insulin signalling can be transduced along metabolic pathways involving phosphatidylinositol 3-kinase (PI3K) and AKT or gene regulato$\mathrm{ry} /$ mitogenic pathways involving mitogen-activated protein kinases (MAPKs), where each of these steps constitutes a critical node in the regulation of metabolic and gene regulatory events controlling insulin sensitivity [1]. Further complexity is built into the system by the expression of multiple isoforms of substrates and protein kinases at each of these nodes. Negative regulation of insulin signalling can 


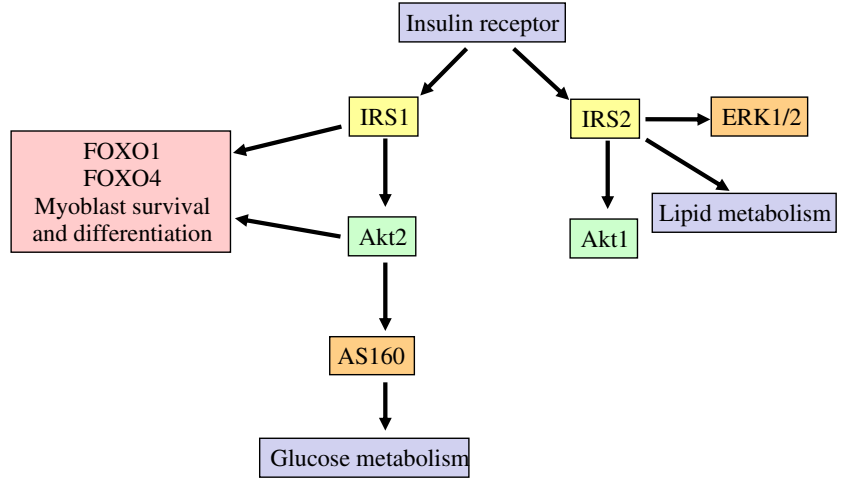

Fig. 1 siRNA-mediated gene silencing reveals specificity in insulin signalling. Insulin signalling pathways involving IRS1 and AKT2 are required for myotube formation, phosphorylation of AKT and AS160 and glucose uptake. Conversely, insulin signalling pathways involving IRS2 are necessary for lipid uptake and metabolism

be conferred through activation of cell-surface receptor signalling pathways that are responsive to cytokines such as TNF- $\alpha$ or IL- 6 , which are produced by macrophages that infiltrate adipose tissue in obesity, as well as to elevated levels of glucose or lipids $[3,4]$. Negative regulation of the insulin cascade occurs through serine phosphorylation events at the level of the insulin receptor [4] and IRS isoforms [5]. Several serine/threonine protein kinases have been implicated in the negative regulation of insulin signalling, including protein kinase $\mathrm{C}$ isoforms, MAPK isoforms, mammalian target of rapamycin (mTOR) and p70 ribosomal S6 kinase (p70S6K) [1].

Small interfering RNA (siRNA) has become widely considered as an essential molecular tool for the validation of genes that could potentially constitute the pivotal nodes in the regulation of cellular metabolism. siRNA can be used to identify critical components of the insulin signalling cascade and to suppress targets that relieve inhibitory inputs to enhance insulin signalling to metabolic endpoints and normalise glucose homeostasis. Here we will review current efforts from our laboratory related to the application of siRNA to elucidate the molecular mechanisms that exert the effects of insulin action on metabolism. We will also consider the use of siRNA to identify and validate targets that prevent insulin resistance. Employing siRNA to unravel the molecular mechanism(s) regulating glucose and lipid metabolism in tissues of human origin will aid attempts to develop diabetes prevention and treatment strategies.

\section{SiRNA: molecular scissors to dissect mechanisms in metabolic disease}

Cellular metabolism can be controlled by the production and activity of numerous proteins that relay critical information to regulate glucose and lipid metabolism and protein production. The message to synthesise new proteins is conveyed by mRNA. In 1998, Fire et al. [6] discovered a novel mechanism of gene silencing by double-stranded RNA, which they termed RNA interference. The RNA is cleaved by the enzyme Dicer into duplexes 21 and 23 nucleotides in length called siRNA, and the siRNA is incorporated into a protein complex that recognises and cleaves target mRNA. The fundamental principles behind siRNA and the strategy involved (Fig. 2) have been extensively reviewed [7].

siRNA has great potential to resolve the complex insulin signalling cascades that control glucose and lipid metabolism in peripheral tissues in type 2 diabetes. Since siRNA can be readily applied to cell-based culture systems, researchers can rapidly validate target genes in liver, skeletal muscle and adipose tissue - organs controlling whole body insulin sensitivity. The advantages of siRNA include that it (1) transiently eliminates specific proteins in fully differentiated cells; (2) rapidly silences gene expression such that any induction of compensatory alternative pathways is minimised; (3) circumvents confounding effects of metabolic derangements and organ-to-organ

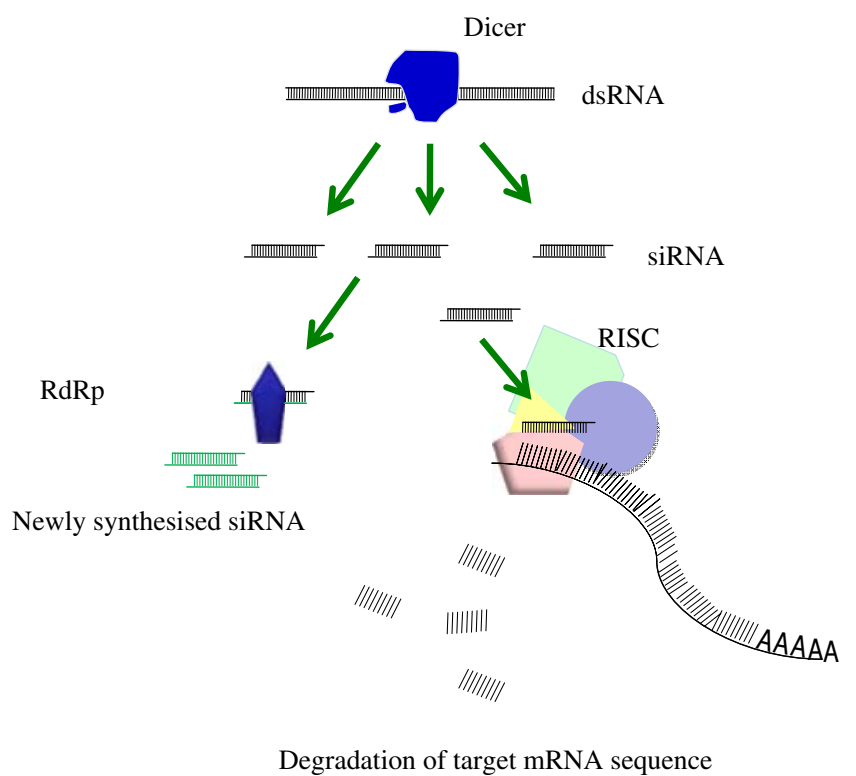

Fig. 2 Mechanism of siRNA gene silencing. Double-stranded (ds) RNA is recognised within the cell by the enzyme Dicer, leading to the generation of siRNA species. In experimental analysis, specific siRNA sequences are usually directly added, leading to the recruitment and assembly of the RNA-induced silencing complex (RISC), which facilitates the targeting and pairing of siRNA to complementary sequences in mRNA. This leads to the degradation of the mRNA, thus reducing the translation of the protein encoded by the mRNA. siRNA may also be replicated and amplified in the cell via the RNAdependent RNA polymerase (RdRp), thus producing a sustained cellular presence of the siRNA 
cross-talks observed in whole body model systems; and (4) can be applied to cultured human tissues.

\section{Targeting insulin signalling cascades using siRNA}

Several reviews have highlighted the critical components of the canonical insulin signalling cascade in health and metabolic disease $[1,2,4,8,9]$. Major nodes, or points of convergence, for insulin signalling include the insulin receptor/IRS1-4, PI3K and its regulatory and catalytic subunits, and AKT/protein kinase B isoforms 1-3 [1]. The $\mathrm{PI} 3 \mathrm{~K} / \mathrm{AKT}$ module is mainly responsible for insulinstimulated metabolic responses, such as increased glucose uptake and protein synthesis, while the MAPK pathway regulates gene expression to control growth and differentiation. Given the important role of skeletal muscle in maintaining whole body insulin sensitivity $[10,11]$, coupled with the fact that skeletal muscle insulin resistance is an early defect in the pathogenesis of type 2 diabetes [12], we have focused on the role of insulin signalling in primary human skeletal muscle. Specifically, our goal is to identify impairments that contribute to the development of skeletal muscle insulin resistance in humans.

Earlier work from our laboratory provides evidence that insulin signalling at the level of IRS1 and IRS2 is impaired in skeletal muscle from type 2 diabetic patients [13, 14], and that this occurs concomitantly with impaired glucose and lipid metabolism [9]. AKT has been implicated in the regulation of glucose transporter translocation and glucose transport [15-17], and some [14, 18-20], but not all [21, 22], studies provide evidence that insulin signalling at the level of AKT is impaired in skeletal muscle from type 2 diabetic patients. Clearly, a detailed molecular analysis of the different effects of the specific IRS or AKT isoforms on metabolic and gene regulatory responses in humans poses a challenge. The greatest insight to date in this regard has been provided by transgenic and knockout mouse models of diabetes. siRNA permits the systematic dissection of isoform-specific and the determination of the impact of these genes on the development of insulin resistance.

Several investigators have used cell-based models and knockout mouse models to dissect the role of IRS and AKT isoforms on skeletal muscle glucose and lipid metabolism [23-29]. Subtle differences in domain structure could confer different roles for IRS1 and IRS2. Although structurally very similar, only IRS2 possesses a kinase regulatory loop binding (KRLB) domain [30, 31]. Mutations resulting in a loss of KRLB binding to the insulin receptor leads to increased insulin-stimulated tyrosine phosphorylation of IRS2 [32], indicating that this domain serves to dampen insulin-mediated activation of IRS2 and, hence, IRS1 is the primary substrate of the activated insulin receptor [32].
To deconvolute insulin signalling cascades controlling positive signals that regulate glucose and lipid metabolism, we used siRNA directed against the major IRS proteins expressed in human skeletal muscle, namely, IRS1 and IRS2 [23]. IRS3 was excluded from our analysis because in silico screening of the human IRS3 genome region, and other biological and molecular findings, provide evidence that humans do not possess a functional IRS3 protein [33]. We also silenced $A K T 1$ and $A K T 2$ in primary human muscle cells. The absence of IRS3 [33] and the report of low or undetectable levels of AKT3 [34] in human muscle provide a rationale for the requirement to validate the critical components of the insulin signalling cascade in human tissue since in rodents, IRS3 or AKT3 may compensate for the deletion or impairment in either IRS1/ IRS2 or AKT1/AKT2, respectively.

siRNA reveals differential signalling along the AKT and MAPK pathways Targeted depletion of IRS1 in primary human myotubes abolished insulin-mediated phosphorylation of AKT Ser473, the AKT substrate of $160 \mathrm{kDa}$ (AS160) and FOXO1/FOXO4, and partly decreased AKT Thr308 phosphorylation, whereas targeted depletion of IRS2 in primary human myotubes abolished insulin action on extracellular signal-regulated kinase $1 / 2$ (ERK1/2) MAPK phosphorylation [34]. This finding of differential signalling responses would suggest that IRS1 relays signals to the metabolic arm of the insulin signalling cascade, whereas IRS2 relays signals along gene regulatory pathways. Our results in human myotubes are consistent with earlier studies in L6 muscle cells [27, 35], in which siRNA directed against either Irs 1 or Irs 2 reduced insulin action on AKT phosphorylation and siRNA targeting Irs 2 reduced insulin action on ERK1/2 MAPK. However, in L6 myotubes, the link between AKT activation and IRS isoforms is less clear. In one study [27], depletion of IRS1 reduced AKT1 and AKT2 phosphorylation, whereas depletion of IRS2 reduced only AKT2 phosphorylation. Conversely, in another study [35], IRS2, not IRS1, was shown to be the primary mediator of insulin-stimulated AKT phosphorylation. The reason for the differences between these studies is unclear; however, the introduction of siRNA into a cell may also have an unknown 'off-target' effect that influences cellular signalling and metabolism above and beyond any effect associated with the gene of interest.

In cultured human myotubes, IRS1 signals to both AKT1 and AKT2, whereas IRS2 signals to ERK1/2 MAPK, thereby indicating isoform specificity in transmitting input between the different nodes of the canonical insulin signalling cascade [23]. This is in agreement with results reported for L6 myotubes using a similar approach [27]. Conversely, in L6 myotubes expressing a mutant 
(Arg1152Gln) insulin receptor originally identified in a patient with severe insulin resistance, insulin-dependent glucose storage and ERK signalling were correlated with IRS2 and IRS1, respectively [36]. Whether overexpression of the mutant Arg1152Gln insulin receptor in these cells alters the relative role of the IRS isoforms is unknown. Overexpression of KRLB peptides in L6 myotubes specifically blocks insulin-mediated IRS2 phosphorylation and increases IRS1 phosphorylation and ERK signalling, while preventing insulin-stimulated glycogen synthesis [37]. Collectively, these findings in L6 myotubes expressing mutant insulin receptors or peptides against the receptor provide evidence suggesting that insulin signalling requires IRS1 for mitogenic effects and IRS2 for glucose metabolism and are therefore not consistent with the results from siRNA-based approaches [23, 27]. The reason for these differences is unclear.

In intact human skeletal muscle, insulin action on both IRS1 and IRS2 is impaired in type 2 diabetic patients, and this occurs concomitantly with reduced PI3K activity but normal ERK1/2 MAPK phosphorylation [14, 38]. One possible explanation is that a maximal effect of insulin on IRS2 phosphorylation is not required for the full effect on ERK1/2 MAPK phosphorylation. Alternatively, signalling events may be organised spatially such that IRS2 phosphorylation in a specialised compartment of the cell may be intact, despite a reduction in total cellular IRS2 signalling.

siRNA reveals specificity in glucose and lipid metabolism The molecular dissection of the critical components of the insulin signalling cascades that regulate distinct metabolic pathways such as glucose or lipid metabolism has been challenging. While knockout mouse models can be used to attempt to identify key proteins, the effect of the absence of a particular gene on early developmental changes may influence the phenotype and, consequently, the whole body metabolic response of the animal, confounding the results. Indeed, an important role of insulin signalling in early development and growth has long been appreciated [33, 34]. Moreover, a recent study in zebrafish provides evidence that insulin receptor signalling is essential for the proper development of brain, heart and eye, and embryonic growth [39]. Human cell culture models offer a complementary approach for investigating the specificity of insulin receptor signalling on metabolic responses that excludes the possibility of any effect of early developmental changes or organ-to-organ communication. To this end, we determined the effect of siRNA silencing of IRSI/IRS2 or $A K T 1 / A K T 2$ on glucose and lipid metabolism in differentiated human myotubes, reporting evidence that basal glucose uptake and metabolism is mediated by IRS2, whereas the insulin-stimulated response requires IRS1 [23], consistent with findings in L6 muscle cells [27]. Furthermore, we found that the action of insulin on glucose uptake was prevented in primary human myotubes depleted of AKT2 but was preserved in myotubes depleted of AKT1 [23]. Reduced levels of AKT2 in 3T3-L1 adipocytes or L6 myotubes [27, 40] attenuates insulin-stimulated glucose transport. Collectively, these results indicate that a signalling module comprising IRS1 and AKT2 controls glucose uptake and metabolism, whereas a module consisting of IRS2 and AKT1 controls lipid oxidation. As indicated earlier, insulin resistance at the level of IRS1 and IRS2 is evident in severe insulin resistance and long-standing type 2 diabetes [13, 14], together with impaired glucose and lipid metabolism [9]. Moreover, in type 2 diabetic patients and obese insulin-resistant humans, impaired AKT2 activity is associated with defects in glucose transport [14, 18-20]. Whether alterations in insulin signalling are a cause or a consequence of impaired whole body glucose homeostasis is unknown, but this modular regulation of distinctive metabolic responses by the insulin signalling cascade offers potential targets for the treatment of defects in glucose or lipid metabolism for the clinical management of type 2 diabetes.

\section{Enhancing insulin action by targeting signalling cascades}

An innovative approach used to attempt to unravel critical components of the insulin signalling cascade involved in the regulation of glucose transport involves the use of siRNA. Presumably, this approach could be used for the whole genome or for selective families of genes, or 'target classes', such as nuclear receptors, G protein-coupled receptors, phosphatases, proteases, ion channels and transporters. For example, an RNA interference-based screen of protein kinases expressed in 3T3-L1 adipocytes has been used to measure the effects of siRNA gene silencing on basal and insulin-stimulated glucose transport [41]. This approach is of clinical relevance given that glucose transport defects, either as a result of reductions in levels of GLUT4 protein, as observed in adipocytes, or impairments in insulin-stimulated GLUT4 translocation, as observed in skeletal muscle and adipocytes, contribute to the whole body impairment in glucose homeostasis in type 2 diabetic patients [9]. However, one of the problems associated with siRNA-based approaches is the possibility that the expression of non-targeted genes may also be altered. siRNA sequences are processed within cells in a manner analogous to endogenous micro RNAs, which, in human tissues, have been shown to affect sequences that only partially match $[42,43]$. Thus, results obtained using siRNA should be validated using several different siRNA 
sequences targeting the same gene and biochemically confirmed in different model systems.

Czech and co-workers [41] performed an siRNA screen to test the hypothesis that suppression of individual protein kinases would identify regulators of cellular GLUT4 protein content and/or membrane trafficking. Initially, gene expression profiling was used to identify all protein kinases present in 3T3-L1 adipocytes, and a high-throughput screening approach was then used to systematically silence the targets using siRNA. As positive controls for the assay, two known positive and negative regulators of glucose transport, Akt2 and Pten, respectively, were silenced [23, 27, 40, 44]. Screening the 58 protein kinases identified in 3T3-L1 adipocytes revealed that silencing the genes encoding the following protein kinases enhanced glucose transport, suggesting that they are endogenous suppressors of glucose transport: the cyclin-dependent kinase-related protein kinases, PCTAIRE-motif protein kinase 1 and PFTAIRE protein kinase 1 , the inhibitor of nuclear factor$\mathrm{KB}$ kinase isoforms $\alpha$ and $\beta$ (IKK $\alpha$ and IKK $\beta$ ), a member of the Sterile 20 family of protein kinases, mitogenactivated protein kinase kinase kinase kinase 4 (MAP4K4, also referred to as NCK-interacting kinase). In contrast, the silencing of the gene encoding integrin-linked protein kinase impaired glucose transport, suggesting that this protein kinase is an endogenous enhancer of glucose transport. Clearly this is a powerful approach for the identification of novel signal transducers that regulate glucose transport. Conceivably, this approach could be adapted to identify new points of regulation of cellular lipid and protein metabolism. The technique could also be applied to models of insulin resistance to identify protein kinases that enhance insulin sensitivity or responsiveness.

\section{Application of siRNA to combat insulin resistance}

Diabetes affects the whole body, and defects in insulinsensitive organs, including skeletal muscle, adipose tissue and liver, can contribute to impairments in whole body glucose homeostasis. Early studies provided clinical evidence that defects in peripheral tissues and in the beta cell are required for the development of type 2 diabetes [12]. The link between peripheral organs and central mechanisms in the control of both glucose and energy homeostasis has also been appreciated for several decades [45-47]. Efforts are under way to apply siRNA to central and peripheral tissues that control glucose and energy homeostasis to improve insulin sensitivity (for reviews see [48, 49]).

For the purposes of this review, two examples will be highlighted to show how specific genes in skeletal muscle can be targeted to prevent the development of insulin resistance. Experimentally, skeletal muscle insulin resis- tance can be induced by exposure to elevated levels of glucose, insulin, NEFA or cytokines such as TNF- $\alpha$. Although these factors induce insulin resistance via different mechanisms and signalling pathways (reviewed in [4]), several of these factors co-exist in vivo and may collectively contribute to the overall clinical phenotype in diabetic patients. For example, an acute infusion of TNF- $\alpha$ into healthy volunteers impairs insulin-mediated skeletal muscle glucose uptake without altering endogenous glucose production [50]. Elevations in TNF- $\alpha$ are correlated with increased phosphorylation of ERK1/2, c-Jun $\mathrm{NH}_{2}$-terminal kinase (JNK) and p70S6K, and increased serine phosphorylation of IRS1 (Fig. 3) [1]. These activated inhibitory molecules exert a negative effect on insulin signalling. TNF- $\alpha$ infusion was also associated with impaired phosphorylation of AS160, the most proximal step identified in the canonical insulin signalling cascade regulating GLUT4 translocation and glucose uptake [50]. Although TNF- $\alpha$ levels are not markedly elevated in lean or moderately obese type 2 diabetic patients [13], in morbidly obese individuals, macrophages infiltrate the adipose tissue and secrete TNF- $\alpha$, creating a low-grade inflammatory state that can contribute to the development of insulin resistance [51]. Based on these lines of evidence, we adopted a cell culture model to induce insulin resistance by exposing primary skeletal muscle myotubes derived from healthy

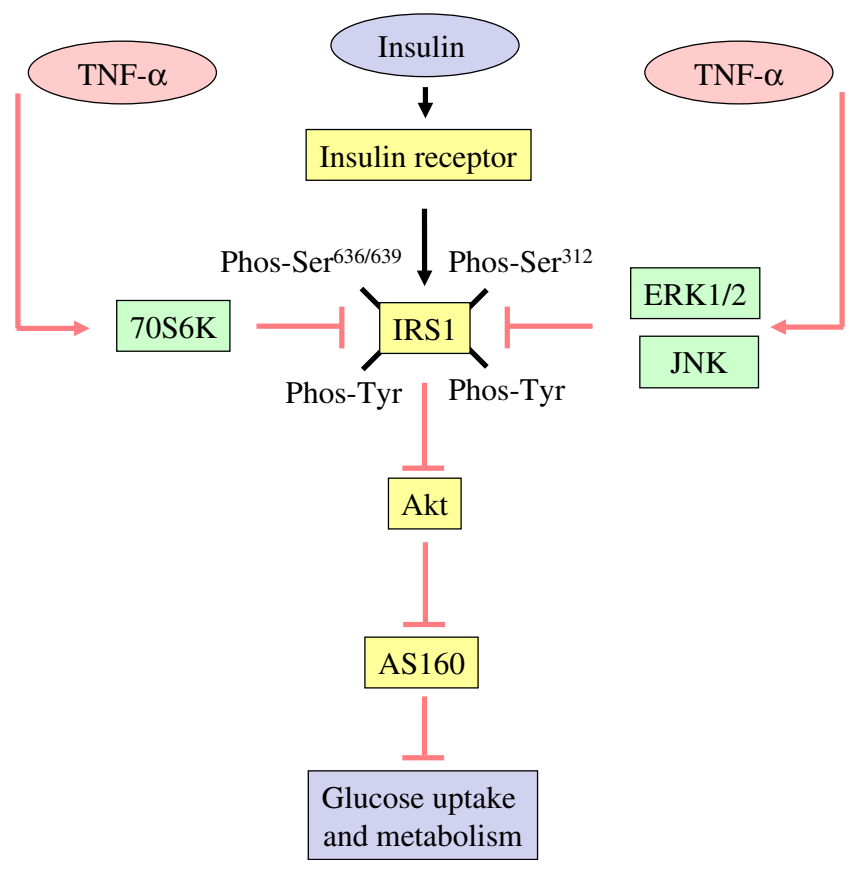

Fig. 3 Effects of TNF- $\alpha$ exposure on insulin signalling. TNF- $\alpha$ exposure increases phosphorylation of p70S6K, ERK1/2, and JNK, concomitant with increased serine phosphorylation of IRS1. These signal transducers act as inhibitory molecules, which provide negative feedback on canonical insulin signalling and impair glucose uptake and metabolism 
volunteers to TNF- $\alpha$. We next used siRNA directed against $I K K \beta$ (also known as $I K B K B$ ) [52] or MAP4K4 [53] - the protein products of which were identified by Czech and coworkers [41] as regulators of cellular GLUT4 protein content and/or membrane trafficking in cultured 3T3-L1 adipocytes - to investigate whether targeted depletion of these proteins would prevent the development of insulin resistance. Recent results from our laboratory related to the role of IKK $\beta$ [52] and MAP4K4 [53] will be reviewed below. Since defects in skeletal muscle glucose transport and cell surface GLUT4 content in type 2 diabetic patients correlate with impaired whole body insulin-mediated glucose uptake [54-56], targeting these novel signalling cascades that regulate GLUT4 or translocation may be efficacious in the treatment of whole body insulin resistance.

$I K K \beta$ The I $\mathrm{KB}$ kinase complex is composed of two catalytic subunits $(\operatorname{IKK} \alpha$ and $\operatorname{IKK} \beta)$ and one regulatory subunit $(\operatorname{IKK} \gamma)$. IKK $\beta$, but not IKK $\alpha$, plays a major role in nuclear factor $\kappa \mathrm{B}(\mathrm{NF}-\mathrm{\kappa B})$ activation and activity in response to proinflammatory cytokines such as TNF- $\alpha$ [57]. In type 2 diabetic patients [58] or lipid-infused healthy volunteers [59], increased IKB/NF- $\mathrm{kB}$ pathway activity is associated with skeletal muscle insulin resistance. Genetic or pharmacological inhibition of the I $\mathrm{kB} / \mathrm{NF}-\mathrm{kB}$ pathway, e.g. by deletion of $I k k \beta$ in mice or by administration of salicylates to a rodent model of diabetes, improves peripheral insulin sensitivity [60]. Salicylates, including aspirin, improve insulin action by inhibiting IKK $\beta$ in animals with obesity- and dietary-induced insulin resistance [61] and prevent the inhibitory effects of TNF- $\alpha$ [62] on insulin signalling.

Using primary human skeletal muscle myotubes we determined the effect of $I K K \beta$ gene silencing by siRNA on insulin action [52]. Myotubes were then incubated in the absence or presence of TNF- $\alpha$ for $2 \mathrm{~h}$. Exposure to TNF- $\alpha$ impaired the action of insulin on glucose uptake and metabolism and reduced the phosphorylation of AKT and the AKT substrate AS160 [52]. Gene silencing reduced IKK $\beta$ protein levels by $75 \%$, without affecting cell differentiation or the expression of GLUT1 or GLUT4 (also known as $S L C 2 A 1$ and $S L C 2 A 4$, respectively) mRNAs. Importantly, $I K K \beta$ gene silencing fully protected against TNF- $\alpha$-induced inhibition of glucose uptake and metabolism and signal transduction at the level of AKT and AS160. Since AS160 plays a critical role in the insulin signalling pathway leading to glucose transport [63], this may in part explain the enhanced glucose metabolism observed in IKK $\beta$-depleted myotubes. These findings provide biological validation of IKK $\beta$ as a potential target for pharmacological interventions aimed at improving insulin sensitivity, highlighting the opportunity for the development readily available over-the-counter pharma- ceuticals, such as aspirin and similar derivatives, for the effective treatment of insulin resistance. Remarkably, blood glucose, total cholesterol, C-reactive protein, triacylglycerol and insulin clearance are improved in type 2 diabetic patients following 2 weeks of treatment with relatively high doses of aspirin (approximately $7 \mathrm{~g} /$ day) [64]. Although the high doses of salicylates required indicate that more research is required to refine putative compounds, this clinical investigation also revealed that the short-term aspirin treatment improved glucose and NEFA levels during a mixed meal tolerance test, as well as reducing basal hepatic glucose production and enhancing peripheral insulin sensitivity during a clamp study. Nevertheless, these currently available over-the-counter drugs have undesired side effects and may therefore prove to be an unreasonable treatment option for insulin resistance. Collectively, the evidence from cultured cells, animal models and clinical investigation underscores IKK $\beta$ as a potential therapeutic target for the prevention of peripheral insulin resistance in the setting of obesity with low-grade inflammation.

MAP4K4 JNK is a widely recognised TNF- $\alpha$-responsive target that negatively regulates insulin signalling to metabolic endpoints [65]. Knockout mice that lack expression of Jnk1 (also known as Mapk8) are protected against the development of dietary-induced obesity and insulin resistance [66]. Thus, suppressors of JNK signalling may enhance insulin action and prevent defects in glucose metabolism. JNK is activated via MAP4K4 signalling in 3T3-L1 adipocytes following treatment with TNF- $\alpha$ [41]. TNF- $\alpha$ receptor 1 (TNFR1), but not TNFR2, mediates an increase in cellular MAP4K4 protein content, and this is in addition to the potent effect of TNF- $\alpha$ on the phosphorylation of JNK and p38 MAPK [67]. In light of the discovery that deletion of MAP4K4 in 3T3-L1 adipocytes restores cellular GLUT4 protein content and trafficking after TNF- $\alpha$ stimulation [41], we examined the role of this protein kinase in human skeletal muscle. Consistent with findings in 3T3-L1 adipocytes, MAP4K4 silencing prevents TNF- $\alpha$-induced JNK and ERK phosphorylation, as well as IRS1 serine phosphorylation [53], and relieves inhibitory signals on AKT and AS160 to promote glucose uptake. Silencing $M A P 2 K 1$ and $M A P 2 K 4$, intermediary kinases involved in ERK and JNK signalling, respectively, also prevented TNF- $\alpha$-induced insulin resistance, which validates these protein kinases as downstream targets of MAP4K4.

Defects in insulin-stimulated glucose transport observed in skeletal muscle from people with type 2 diabetes persist in cultured myotubes [68], indicating that intrinsic defects influence insulin action. Thus, cultured myotubes derived from skeletal muscle biopsies from type 2 diabetic patients represent a suitable cellular model to validate whether gene silencing of candidate negative regulators of insulin 
signalling boosts metabolism. We transfected myotubes from type 2 diabetic patients and volunteers with normal glucose tolerance to determine whether MAP4K4 silencing improves insulin action in diabetes [53]. Silencing of $M A P 4 K 4, M A P 2 K 1$ or MAP2K4 completely restored insulin action on glucose uptake. Thus, strategies to inhibit MAP4K4 signalling are efficacious in the prevention of inhibitory signals that cause insulin resistance in skeletal muscle.

\section{Conclusion}

siRNA is a powerful tool for the validation of specific targets controlling metabolism and gene regulatory events in health and disease. Future efforts using an unbiased approach to systematically silence all expressed genes in a specific tissue may reveal novel modes of metabolic regulation. With high-throughput assay systems, the complexity of signalling pathways can be rapidly deconvoluted. Several negative regulators of glucose uptake and metabolism have already been revealed by high-throughput analysis of glucose transport and GLUT4 translocation in cultured cells [69]. This same approach can be taken to identify targets that protect against the development of insulin resistance. For example, suppression of PTEN, $P T P 1 B, J N K 1, p 70 S 6 K$ or $P K C \theta$ (also known as PRKCQ) using siRNA protects against palmitate-induced insulin resistance [69] and, consequently, may hold promise as therapeutic targets to pharmacologically enhance insulin action in type 2 diabetes.

Future efforts will require a combined approach with high-throughput assay systems, physiologically relevant secondary cell culture systems, animal models with tissuespecific gene knockout and clinically orientated investigations to reveal a three-dimensional view of the integrated biology controlling tissue-specific and whole body glucose homeostasis. Biased and unbiased approaches will be required whereby siRNA against known genes, or specially designed target libraries, will be useful to validate the pathways that govern the actions of insulin on glucose and lipid metabolism. The specificity inherent in siRNA has spurred efforts to translate the basic science in this area into clinical applications [70]. While the development of siRNA-based therapy remains promising, there are still many obstacles to overcome. Indeed, siRNA has sequenceand target-independent effects on gene expression that may have a negative impact in terms of compromising safety in the clinic. Furthermore, the potential therapeutic application of siRNA will require effective delivery systems to target cells and tissues, which may present an additional challenge for the treatment of metabolic disease. While the translational application of siRNA to treat insulin resistance in humans is still outside our reach, strategies to both mitigate off-target effects and advance siRNA delivery are currently being developed.

Acknowledgements Funding has been provided by the Swedish Research Council (A. Krook and J. R. Zierath), Swedish Foundation for Strategic Research (J. R. Zierath) and the European Research Council (J. R. Zierath).

Duality of interest The authors declare that there is no duality of interest associated with this manuscript.

\section{References}

1. Taniguchi CM, Emanuelli B, Kahn CR (2006) Critical nodes in signalling pathways: insights into insulin action. Nat Rev Mol Cell Biol 7:85-96

2. White MF (2002) IRS proteins and the common path to diabetes. Am J Physiol Endocrinol Metab 283:E413-E422

3. Guilherme A, Virbasius JV, Puri V, Czech MP (2008) Adipocyte dysfunctions linking obesity to insulin resistance and type 2 diabetes. Nat Rev Mol Cell Biol 9:367-377

4. Pirola L, Johnston AM, Van Obberghen E (2004) Modulation of insulin action. Diabetologia 47:170-184

5. Boura-Halfon S, Zick Y (2008) Phosphorylation of IRS proteins, insulin action and insulin resistance. Am J Physiol Endocrinol Metab. doi:10.1152/ajpendo.90437.2008

6. Fire A, Xu S, Montgomery MK, Kostas SA, Driver SE, Mello CC (1998) Potent and specific genetic interference by double-stranded RNA in Caenorhabditis elegans. Nature 391:806-811

7. Lehner B, Fraser AG, Sanderson CM (2004) Technique review: how to use RNA interference. Brief Funct Genomic Proteomic $3: 68-83$

8. Khan AH, Pessin JE (2002) Insulin regulation of glucose uptake: a complex interplay of intracellular signalling pathways. Diabetologia 45:1475-1483

9. Zierath JR, Krook A, Wallberg-Henriksson H (2000) Insulin action and insulin resistance in human skeletal muscle. Diabetologia 43:821-835

10. DeFronzo RA, Gunnarsson R, Bjorkman O, Olsson M, Wahren J (1985) Effects of insulin on peripheral and splanchnic glucose metabolism in noninsulin-dependent (type II) diabetes mellitus. J Clin Invest 76:149-155

11. Taylor R (2008) Pathogenesis of type 2 diabetes: tracing the reverse route from cure to cause. Diabetologia 51:1781-1789

12. Eriksson J, Franssila-Kallunki A, Ekstrand A et al (1989) Early metabolic defects in persons at increased risk for non-insulindependent diabetes mellitus. N Engl J Med 321:337-343

13. Bjornholm M, Kawano Y, Lehtihet M, Zierath JR (1997) Insulin receptor substrate-1 phosphorylation and phosphatidylinositol 3kinase activity in skeletal muscle from NIDDM subjects after in vivo insulin stimulation. Diabetes 46:524-527

14. Krook A, Bjornholm M, Galuska D et al (2000) Characterization of signal transduction and glucose transport in skeletal muscle from type 2 diabetic patients. Diabetes 49:284-292

15. Kohn AD, Summers SA, Birnbaum MJ, Roth RA (1996) Expression of a constitutively active Akt Ser/Thr kinase in 3T3L1 adipocytes stimulates glucose uptake and glucose transporter 4 translocation. J Biol Chem 271:31372-31378

16. Ueki K, Yamamoto-Honda R, Kaburagi Y et al (1998) Potential role of protein kinase B in insulin-induced glucose transport, glycogen synthesis, and protein synthesis. J Biol Chem 273:53155322 
17. Wang Q, Somwar R, Bilan PJ et al (1999) Protein kinase B/Akt participates in GLUT4 translocation by insulin in L6 myoblasts. Mol Cell Biol 19:4008-4018

18. Krook A, Roth RA, Jiang XJ, Zierath JR, Wallberg-Henriksson H (1998) Insulin-stimulated Akt kinase activity is reduced in skeletal muscle from NIDDM subjects. Diabetes 47:1281-1286

19. Brozinick JT Jr, Roberts BR, Dohm GL (2003) Defective signaling through Akt-2 and -3 but not Akt-1 in insulin-resistant human skeletal muscle: potential role in insulin resistance. Diabetes 52:935-941

20. Gosmanov AR, Umpierrez GE, Karabell AH, Cuervo R, Thomason DB (2004) Impaired expression and insulin-stimulated phosphorylation of Akt-2 in muscle of obese patients with atypical diabetes. Am J Physiol Endocrinol Metab 287:E8-E15

21. Kim YB, Nikoulina SE, Ciaraldi TP, Henry RR, Kahn BB (1999) Normal insulin-dependent activation of Akt/protein kinase B, with diminished activation of phosphoinositide 3-kinase, in muscle in type 2 diabetes. J Clin Invest 104:733-741

22. Beeson M, Sajan MP, Dizon M et al (2003) Activation of protein kinase $\mathrm{C}-\zeta$ by insulin and phosphatidylinositol-3,4,5-( $\left(\mathrm{PO}_{4}\right)_{3}$ is defective in muscle in type 2 diabetes and impaired glucose tolerance: amelioration by rosiglitazone and exercise. Diabetes 52:1926-1934

23. Bouzakri K, Zachrisson A, Al-Khalili L et al (2006) siRNA-based gene silencing reveals specialized roles of IRS-1/Akt2 and IRS-2/ Akt1 in glucose and lipid metabolism in human skeletal muscle. Cell Metab 4:89-96

24. Cho $\mathrm{H}, \mathrm{Mu} \mathrm{J}$, Kim JK et al (2001) Insulin resistance and a diabetes mellitus-like syndrome in mice lacking the protein kinase Akt2 (PKB $\beta)$. Science 292:1728-1731

25. Cho H, Thorvaldsen JL, Chu Q, Feng F, Birnbaum MJ (2001) $\mathrm{Akt} 1 / \mathrm{PKB} \alpha$ is required for normal growth but dispensable for maintenance of glucose homeostasis in mice. J Biol Chem 276:38349-38352

26. Higaki Y, Wojtaszewski JF, Hirshman MF et al (1999) Insulin receptor substrate-2 is not necessary for insulin- and exercisestimulated glucose transport in skeletal muscle. J Biol Chem 274:20791-20795

27. Huang C, Thirone AC, Huang X, Klip A (2005) Differential contribution of insulin receptor substrates 1 versus 2 to insulin signaling and glucose uptake in 16 myotubes. J Biol Chem 280:19426-19435

28. Araki E, Lipes MA, Patti ME et al (1994) Alternative pathway of insulin signalling in mice with targeted disruption of the IRS-1 gene. Nature 372:186-190

29. Withers DJ, Gutierrez JS, Towery H et al (1998) Disruption of IRS-2 causes type 2 diabetes in mice. Nature 391:900-904

30. Sawka-Verhelle D, Baron V, Mothe I, Filloux C, White MF, Van Obberghen E (1997) Tyr624 and Tyr628 in insulin receptor substrate- 2 mediate its association with the insulin receptor. J Biol Chem 272:16414-16420

31. Sawka-Verhelle D, Tartare-Deckert S, White MF, Van Obberghen E (1996) Insulin receptor substrate-2 binds to the insulin receptor through its phosphotyrosine-binding domain and through a newly identified domain comprising amino acids 591-786. J Biol Chem 271:5980-5983

32. Wu J, Tseng YD, Xu C-F, Neubert TA, White MF, Hubbard SR (2008) Structural and biochemical characterization of the KRLB region in insulin receptor substrate-2. Nat Struct Mol Biol $15: 251-258$

33. Bjornholm M, He AR, Attersand A et al (2002) Absence of functional insulin receptor substrate-3 (IRS-3) gene in humans. Diabetologia 45:1697-1702

34. Bouzakri K, Roques M, Debard C et al (2004) WY-14643 and 9-cis-retinoic acid induce IRS-2/PI 3-kinase signalling pathway and increase glucose transport in human skeletal muscle cells: differential effect in myotubes from healthy subjects and type 2 diabetic patients. Diabetologia 47:1314-1323

35. Pirola L, Bonnafous S, Johnston AM, Chaussade C, Portis F, Van Obberghen E (2003) Phosphoinositide 3-kinase-mediated reduction of insulin receptor substrate- $1 / 2$ protein expression via different mechanisms contributes to the insulin-induced desensitization of its signaling pathways in L6 muscle cells. J Biol Chem 278:15641-15651

36. Miele C, Caruso M, Calleja V et al (1999) Differential role of insulin receptor substrate (IRS)-1 and IRS-2 in L6 skeletal muscle cells expressing the Arg1152 $\rightarrow$ Gln insulin receptor. J Biol Chem 274:3094-3102

37. Oriente F, Formisano P, Miele C et al (2001) Insulin receptor substrate- 2 phosphorylation is necessary for protein kinase $\mathrm{C} \zeta$ activation by insulin in L6hIR Cells. J Biol Chem 276:37109-37119

38. Cusi K, Maezono K, Osman A et al (2000) Insulin resistance differentially affects the PI 3-kinase- and MAP kinase-mediated signaling in human muscle. J Clin Invest 105:311-320

39. Toyoshima Y, Monson C, Duan C et al (2008) The role of insulin receptor signaling in zebrafish embryogenesis. Endocrinology 149:5996-6005

40. Jiang ZY, Zhou QL, Coleman KA, Chouinard M, Boese Q, Czech MP (2003) Insulin signaling through Akt/protein kinase B analyzed by small interfering RNA-mediated gene silencing. Proc Natl Acad Sci U S A 100:7569-7574

41. Tang X, Guilherme A, Chakladar A et al (2006) An RNA interference-based screen identifies MAP4K4/NIK as a negative regulator of PPAR $\gamma$, adipogenesis, and insulin-responsive hexose transport. Proc Natl Acad Sci U S A 103:2087-2092

42. Ambros V (2004) The functions of animal microRNAs. Nature 431:350-355

43. Ambros V, Chen X (2007) The regulation of genes and genomes by small RNAs. Development 134:1635-1641

44. Tang X, Powelka AM, Soriano NA, Czech MP, Guilherme A (2005) PTEN, but not SHIP2, suppresses insulin signaling through the phosphatidylinositol 3-kinase/Akt pathway in 3T3L1 adipocytes. J Biol Chem 280:22523-22529

45. Coleman DL, Hummel KP (1969) Effects of parabiosis of normal with genetically diabetic mice. Am J Physiol 217:1298-1304

46. Coleman DL, Hummel KP (1970) The effects of hypothalamics lesions in genetically diabetic mice. Diabetologia 6:263-267

47. Hervey GR (1959) The effects of lesions in the hypothalamus in parabiotic rats. J Physiol 145:336-352

48. Rondinone CM (2006) Therapeutic potential of RNAi in metabolic diseases. Biotechniques (Suppl):31-36

49. Kolfschoten IG, Regazzi R (2007) Technology insight: small, noncoding RNA molecules as tools to study and treat endocrine diseases. Nat Clin Pract Endocrinol Metab 3:827-834

50. Plomgaard P, Bouzakri K, Krogh-Madsen R, Mittendorfer B, Zierath JR, Pedersen BK (2005) Tumor necrosis factor- $\alpha$ induces skeletal muscle insulin resistance in healthy human subjects via inhibition of Akt substrate 160 phosphorylation. Diabetes 54: 2939-2945

51. Schenk S, Saberi M, Olefsky JM (2008) Insulin sensitivity: modulation by nutrients and inflammation. J Clin Invest 118:2992-3002

52. Austin RL, Rune A, Bouzakri K, Zierath JR, Krook A (2008) siRNA-mediated reduction of inhibitor of nuclear factor- $\mathrm{kB}$ kinase prevents tumor necrosis factor- $\alpha$-induced insulin resistance in human skeletal muscle. Diabetes 57:2066-2073

53. Bouzakri K, Zierath JR (2007) MAP4K4 gene silencing in human skeletal muscle prevents tumor necrosis factor-alpha-induced insulin resistance. J Biol Chem 282:7783-7789

54. Koistinen HA, Galuska D, Chibalin AV et al (2003) 5-Aminoimidazole carboxamide riboside increases glucose transport and cell-surface GLUT4 content in skeletal muscle from subjects with type 2 diabetes. Diabetes 52:1066-1072 
55. Ryder JW, Yang J, Galuska D et al (2000) Use of a novel impermeable biotinylated photolabeling reagent to assess insulinand hypoxia-stimulated cell surface GLUT4 content in skeletal muscle from type 2 diabetic patients. Diabetes 49:647-654

56. Zierath JR, He L, Guma A, Odegoard Wahlstrom E, Klip A, Wallberg-Henriksson H (1996) Insulin action on glucose transport and plasma membrane GLUT4 content in skeletal muscle from patients with NIDDM. Diabetologia 39:1180-1189

57. Li ZW, Chu W, Hu Y et al (1999) The IKK $\beta$ subunit of IKB kinase (IKK) is essential for nuclear factor $\mathrm{kB}$ activation and prevention of apoptosis. J Exp Med 189:1839-1845

58. Sriwijitkamol A, Christ-Roberts C, Berria R et al (2006) Reduced skeletal muscle inhibitor of $\mathrm{kB} \beta$ content is associated with insulin resistance in subjects with type 2 diabetes: reversal by exercise training. Diabetes 55:760-767

59. Itani SI, Ruderman NB, Schmieder F, Boden G (2002) Lipidinduced insulin resistance in human muscle is associated with changes in diacylglycerol, protein kinase $\mathrm{C}$, and I $\mathrm{kB}-\alpha$. Diabetes 51:2005-2011

60. Shoelson SE, Lee J, Goldfine AB (2006) Inflammation and insulin resistance. J Clin Invest 116:1793-1801

61. Yuan M, Konstantopoulos N, Lee J et al (2001) Reversal of obesity- and diet-induced insulin resistance with salicylates or targeted disruption of Ikk $\beta$. Science 293:1673-1677

62. Jiang G, Dallas-Yang Q, Liu F, Moller DE, Zhang BB (2003) Salicylic acid reverses phorbol 12-myristate-13-acetate (PMA)and tumor necrosis factor $\alpha(\mathrm{TNF} \alpha)$-induced insulin receptor substrate 1 (IRS1) serine 307 phosphorylation and insulin resistance in human embryonic kidney 293 (HEK293) cells. J Biol Chem 278:180-186

63. Sano H, Kane S, Sano E et al (2003) Insulin-stimulated phosphorylation of a Rab GTPase-activating protein regulates GLUT4 translocation. J Biol Chem 278:14599-14602

64. Hundal RS, Petersen KF, Mayerson AB et al (2002) Mechanism by which high-dose aspirin improves glucose metabolism in type 2 diabetes. J Clin Invest 109:1321-1326

65. Weston CR, Davis RJ (2007) The JNK signal transduction pathway. Curr Opin Cell Biol 19:142-149

66. Hirosumi J, Tuncman G, Chang L et al (2002) A central role for JNK in obesity and insulin resistance. Nature 420:333-336

67. Tesz GJ, Guilherme A, Guntur KV et al (2007) Tumor necrosis factor $\alpha(\mathrm{TNF} \alpha)$ stimulates Map4k4 expression through TNF $\alpha$ receptor 1 signaling to c-Jun and activating transcription factor 2 . J Biol Chem 282:19302-19312

68. Henry RR, Abrams L, Nikoulina S, Ciaraldi TP (1995) Insulin action and glucose metabolism in nondiabetic control and NIDDM subjects. Comparison using human skeletal muscle cell cultures. Diabetes 44:936-946

69. Liu F, Dallas-Yang Q, Castriota G et al (2008) Development of a novel GLUT4 translocation assay for identifying potential novel therapeutic targets for insulin sensitization. Biochem J 418:413420

70. Castanotto D, Rossi JJ (2009) The promises and pitfalls of RNAinterference-based therapeutics. Nature 457:426-433 